\title{
Implicit expectiles and measures of implied volatility
}

\author{
Fabio Bellini $\quad$ Lorenzo Mercuri $\quad$ Edit Rroji ${ }^{*}$ \\ $\dagger$ Department of Statistics and Quantitative Methods, \\ University of Milano-Bicocca \\ $\ddagger$ Department of Economics, Management and Quantitative Methods, \\ University of Milan, \\ CREST Japan Science and Technology Agency \\ Tokyo Japan
}

May 2, 2018

\begin{abstract}
We show how to compute the expectiles of the risk neutral distribution from the prices of European call and put options. Empirical properties of these implicit expectiles are studied on a dataset of closing daily prices of FTSE MIB index options. We introduce the interexpectile difference $\Delta_{\tau}(X):=e_{\tau}(X)-e_{1-\tau}(X)$, for $\tau \in(1 / 2,1]$, and suggest that it is a natural measure of the variability of the risk neutral distribution.

We investigate its theoretical and empirical properties and compare it with the VIX index computed by CBOE. We also discuss a theoretical comparison with implicit VaR and CVaR introduced in Barone Adesi (2016).
\end{abstract}

Keywords: Implied Volatility; VIX Index; Expectiles; Interexpectile difference.

\section{Introduction}

From the seminal paper of Breeden and Litzenberger (1978) it is well known that the prices of European call or put options across a continuum of strikes completely determine the risk neutral distribution of the underlying asset at maturity. In the literature, a number of indexes has been suggested in order to extract synthetic information from option prices, the most famous ones being the VIX and SKEW indexes computed as described in CBOE (2009) and CBOE (2010). As it is well known, the VIX formula is based on an approximation of the fair value of a variance swap (Demeterfi et al., 1999), and can also be seen as an approximation of the variance of the risk neutral distribution, as shown in Britten-Jones and Neuberger (2000). The impact of truncation errors and of the finiteness of the number of strikes has been examined in Jiang and Tian (2007). The CBOE Skewness Index is based on the fair price of a cubic contract, as derived in Bakshi et al. (2003).

Recently, there has been much interest also in other functionals of the risk neutral distribution. Barone Adesi (2016) suggested to consider implicit Value at Risk and implicit Expected Shortfall, and extensive empirical analysis on S\&P500 Index and WTI crude oil options have been provided in Barone Adesi et al. (2016a) and Barone Adesi et al. (2016b). Elyasiani et al. (2016) introduced a risk-asymmetry index based on the normalized difference of positive and negative corridor implied volatilities and studied its empirical properties on FTSE MIB index options.

*Corresponding author. Email: edit.rroji@unimib.it 
Expectiles are statistical functionals introduced in Newey and Powell (1987). They are an asymmetric generalization of the mean, arising from the minimization of the expected value of an asymmetric quadratic loss function. Expectiles share many similarities with quantiles, and the expectile curve completely characterizes a distribution, as shown e.g. in Bellini et al. (2016). They are becoming quite popular as risk measures since they are the only example of an elicitable and coherent risk measure (see Bellini and Bignozzi (2015), Bellini and Di Bernardino (2017), Delbaen et al. (2016), and the references therein).

In this paper we show how to compute implicit expectiles from European option prices. It turns out that the computation is very simple and each couple of quoted call and put prices gives an exact point of the inverse expectile curve, that does not depend on any interpolation method.

In order to derive from implicit expectiles a measure of pure variability of the risk neutral distribution that can be compared with the VIX index, we consider the interexpectile difference

$$
\Delta_{\tau}=e_{\tau}(X)-e_{1-\tau}(X),
$$

for $\tau \in(1 / 2,1]$. The interexpectile difference is a natural analogue of the interquantile difference, with the theoretical advantage that it is consistent with the convex order $\leq_{c x}$, in the sense that

$$
X \leq_{c x} Y \Rightarrow \Delta_{\tau}(X) \leq \Delta_{\tau}(Y)
$$

that is a natural requirement for a variability measure.

Our empirical analysis is based on a two-year dataset of closing prices of FTSE MIB Index options. We study the daily dynamic of expectile curves and interexpectile differences, we compare interexpectile differences with a recalculated italian VIX-like index, and finally we consider the forecasting power of the interexpectile difference on future logreturns following the methodology of e.g. Banerjee et al. (2007), Rubbaniy et al. (2014), Elyasiani et al. (2016).

The paper is structured as follows: In Section 2 we discuss the computation of implicit expectiles and we compare them with VIX and implicit VaR and CVaR. In Section 3 we introduce and derive the main properties of the interexpectile difference. In Section 4 we present the results of our empirical analysis on expectile curves and interexpectile differences. Statistical modelling of the interexpectile difference with Lévy Continuous Autoregressive Models is explored in Section 5. In Section 6 we study the relationship between interexpectile differences and future logreturns. Section 7 briefly concludes.

\section{Implicit expectiles}

The expectiles $e_{\tau}$ of a random variable $X \in L^{1}(\Omega, \mathcal{F}, P)$ are a one-parameter family of statistical functionals that shares many similarities with the quantiles. They have been originally defined by Newey and Powell (1987) for $X \in L^{2}(\Omega, \mathcal{F}, P)$ as follows:

$$
e_{\tau}(X):=\underset{x \in \mathbb{R}}{\operatorname{argmin}} \mathbb{E}\left[\tau(X-x)_{+}^{2}+(1-\tau)(X-x)_{-}^{2}\right], \quad \tau \in(0,1) .
$$

Equivalently, they can be defined for any $X \in L^{1}(\Omega, \mathcal{F}, P)$ as the unique solution of the equation

$$
\tau \mathbb{E}\left[\left(X-e_{\tau}(X)\right)_{+}\right]=(1-\tau) \mathbb{E}\left[\left(X-e_{\tau}(X)\right)_{-}\right]
$$

Clearly, $e_{1 / 2}(X)=\mathbb{E}[X]$, so expectiles are a one-parameter family that includes the mean as a special case. We collect their main properties in the following proposition and refer to Newey and Powell (1987), Bellini et al. (2014), Bellini and Di Bernardino (2017) and the references therein for further properties and financial applications.

Proposition 2.1. Let $X \in L^{1}$ and $\tau \in(0,1)$. Then:

a) $e_{\tau}(X+h)=e_{\tau}(X)+h$, for each $h \in \mathbb{R}$ 
b) $e_{\tau}(\lambda X)=\lambda e_{\tau}(X)$, for each $\lambda \geq 0$

c) $X \leq Y$ a.s., $P(X<Y)>0 \Rightarrow e_{\tau}(X)<e_{\tau}(Y)$, for each $\tau \in(0,1)$

d) $e_{\tau}$ is strictly increasing with respect to $\tau$

e) $e_{\tau}$ is continuous with respect to $\tau$

f) $\lim _{\tau \rightarrow 0^{+}} e_{\tau}(X)=\operatorname{essinf}(X), \lim _{\tau \rightarrow 1^{-}} e_{\tau}(X)=\operatorname{esssup}(X)$

g) $e_{\tau}(-X)=-e_{1-\tau}(X)$

h) if $\tau \leq 1 / 2$, then $e_{\tau}(X+Y) \geq e_{\tau}(X)+e_{\tau}(Y)$; if $\tau \geq 1 / 2$, then $e_{\tau}(X+Y) \leq e_{\tau}(X)+e_{\tau}(Y)$

i) if $\tau \leq 1 / 2$, then $X \leq_{c x} Y \Rightarrow e_{\tau}(X) \geq e_{\tau}(Y)$; if $\tau \geq 1 / 2$, then $X \leq_{c x} Y \Rightarrow e_{\tau}(X) \leq e_{\tau}(Y)$,

where $X \leq_{c x} Y$ if $\mathbb{E}[f(X)] \leq \mathbb{E}[f(Y)]$ for each convex $f: \mathbb{R} \rightarrow \mathbb{R}$ such that both expectations exist.

In order to derive a formula for implicit expectiles, we consider a one-period idealized option market where prices of European calls and puts with maturity $T$ are quoted, for all strikes $K \in[0,+\infty)$. We denote with $S_{T}$ the price of the option's underlying asset at maturity, with $r$ the risk-free interest rate, with $C(K)$ and $P(K)$ respectively the prices of call and put options with strike price $K$ and with $\mathbb{Q}$ the risk neutral probability measure, so by risk neutral valuation it holds that

$$
C(K)=e^{-r T} \mathbb{E}_{\mathbb{Q}}\left[\left(S_{T}-K\right)_{+}\right], \quad P(K)=e^{-r T} \mathbb{E}_{\mathbb{Q}}\left[\left(S_{T}-K\right)_{-}\right] .
$$

The definition of expectiles through equation (2) depends only on $\mathbb{E}\left[\left(X-e_{\tau}\right)_{+}\right]$and $\mathbb{E}\left[\left(X-e_{\tau}\right)_{-}\right]$that are given by (3) as prices of European call and put options with strike price $e_{\tau}$. It follows that the implicit $\tau$-expectile is the unique strike price $\bar{K}$ such that

$$
\tau C(\bar{K})=(1-\tau) P(\bar{K}) .
$$

When $\tau=1 / 2$ obviously $\bar{K}$ is equal to the forward price, that is the unique strike price such that $C(\bar{K})=P(\bar{K})$. When $\tau \neq 1 / 2$, implicit expectiles can be seen as forward prices of an asymmetric forward contract, whose payoff (see Figure 1 ) is obtained by buying a quantity $q_{C}$ of call options and selling short a quantity $q_{P}$ of put options such that

$$
\frac{q_{C}}{q_{P}}=\frac{1-\tau}{\tau} .
$$

In an ideal market with a continuum of quoted strikes, equation (4) enables to compute the whole expectile curve $\tau \mapsto e_{\tau}$. Since in real markets call and put prices are quoted on a finite and discrete grid of strikes $K=K_{1}, \ldots, K_{n}$, there are two possible approaches. The first is to interpolate option prices or implied volatilities in order to compute theoretical prices also for strikes with no quotations, in order to be able to solve (4) for each $\tau \in(0,1)$. The second approach is to compute the inverse expectile curve $K \mapsto \tau(K)$ only for quoted strikes, by the formula

$$
\tau\left(K_{i}\right):=\frac{P\left(K_{i}\right)}{P\left(K_{i}\right)+C\left(K_{i}\right)}, \quad i=1, \ldots, n .
$$

Interpolation and extrapolation between quoted implied volatilities has the advantage of producing a continuous curve of implicit expectiles; this is particularly useful if the number of quoted strikes is low, that happens when liquidity is low. The advantage of the second approach is that it is completely nonparametric and does not depend on the choice of an interpolation method; the values $\tau\left(K_{i}\right)$ are exact, insensible to discretization errors. Notice also that formula (5) does not depend on the riskfree 


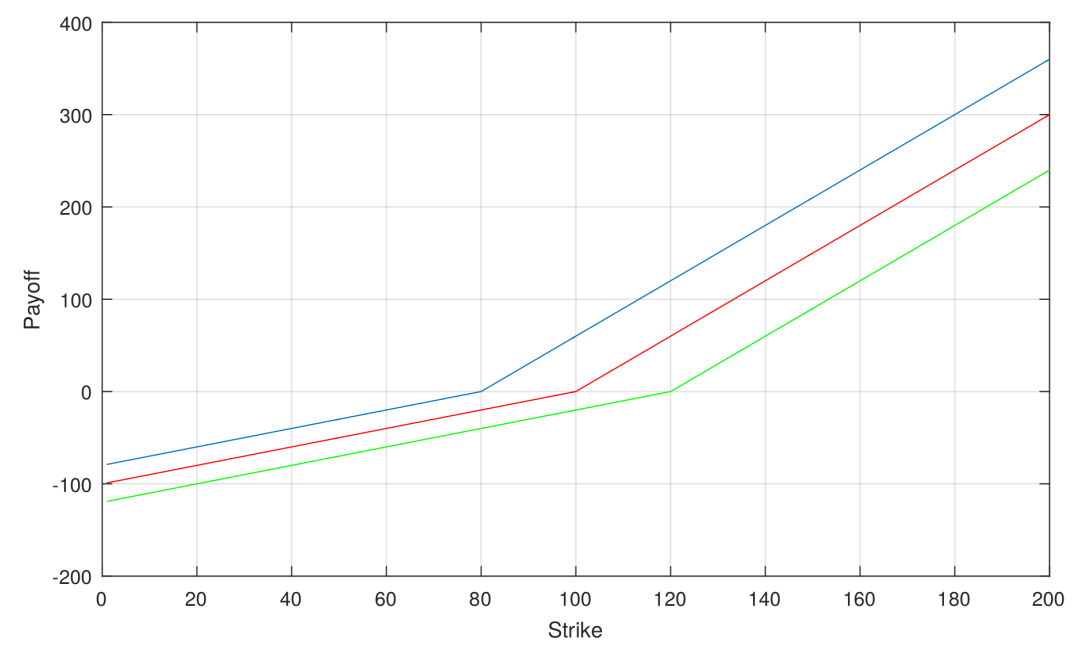

Figure 1: Payoffs of asymmetric forward contracts for $\tau=0.75$ and $K=80,100,120$. The 0.75 implicit expectile is given by the strike for which the price of the payoff is equal to 0 .

rate $r$ or on the dividend yield $d$. If on a given strike only the quotation of the call or of the put is present, the other quotation can be determined by means of the put-call parity and (5) becomes

$$
\tau\left(K_{i}\right)=\frac{C\left(K_{i}\right)-S_{t} e^{-d(T-t)}+K_{i} e^{-r(T-t)}}{2 C\left(K_{i}\right)-S_{t} e^{-d(T-t)}+K_{i} e^{-r(T-t)}} \text { or } \tau\left(K_{i}\right)=\frac{P\left(K_{i}\right)}{2 P\left(K_{i}\right)+S_{t} e^{-d(T-t)}-K_{i} e^{-r(T-t)}} .
$$

Of course in this case the validity of the put-call parity becomes a crucial assumption and implicit expectiles do depend on the riskfree $r$ and on the dividend yield $d$. As an illustration, we compute the inverse expectile curves in the Black-Scholes model, for $S_{t}=30000, r=0, d=0$, and different volatilities and times to maturity. The results are reported in Figure 2. Notice first that since the forward price is equal to 30000 , all the inverse expectile curves have value $1 / 2$ for $K=30000$. Second, the higher the volatility or the time to maturity, the more disperse the risk neutral distribution is, and so the flatter are the inverse expectile curves. Finally, since for the chosen parameters the lognormal risk neutral distributions are almost symmetrical, the same applies to inverse expectile curves.

\subsection{Comparison with other functionals of the risk neutral distribution}

It is interesting to compare implicit expectiles with other well known indexes such as VIX and SKEW computed by CBOE and with the recently introduced implicit VaR and implicit Expected Shortfall (see CBOE (2009), CBOE (2010), Barone Adesi (2016)).

The VIX index is designed to represent a 30-day implied volatility and is computed as the square root of a time-weighted average of the implied variances of the two maturities closer to 30 days. Each implied variance is computed by the formula

$$
\sigma_{T}^{2}=\frac{2}{T} \sum_{i} \frac{\Delta K_{i}}{K_{i}^{2}} e^{r T} O\left(K_{i}\right)-\frac{1}{T}\left(\frac{F}{K_{0}}-1\right)^{2},
$$

where $O\left(K_{i}\right)$ is the price of an OTM call or put with strike $K_{i}, F$ is the forward price, $K_{0}$ is the highest quoted strike lower than $F$. As shown by Demeterfi et al. (1999), this quantity is a discretization of the risk neutral expectation of the realized variance, and it can be written as

$$
\sigma_{T}^{2}=\frac{2 e^{r T}}{T}\left(\int_{0}^{F} \frac{P(K)}{K^{2}} d K+\int_{F}^{+\infty} \frac{C(K)}{K^{2}} d K\right) .
$$



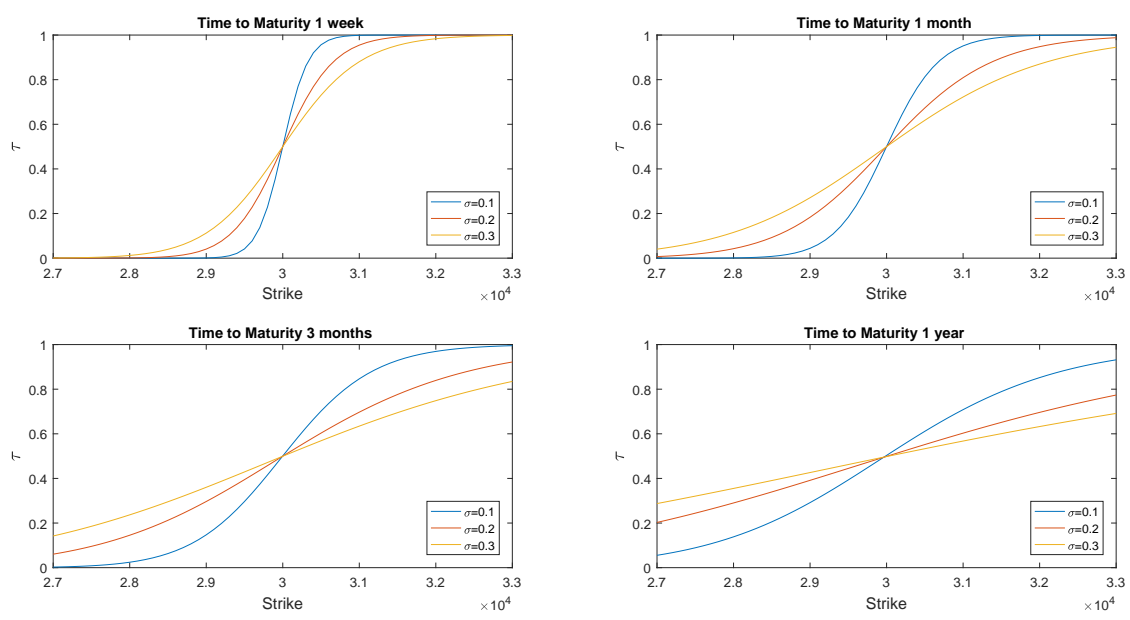

Figure 2: Inverse expectile curves in the Black-Scholes model for annual volatilities $\sigma=10 \%, \sigma=20 \%$, $\sigma=30 \%$ and times to maturity respectively $T-t=1 \mathrm{w}, 1 \mathrm{~m}, 3 \mathrm{~m}, 1 \mathrm{y}$.

VIX is thus equal to the standard deviation of the risk neutral distribution, and is computed by means of all OTM available call and put prices; Fukasawa et al. (2011) showed that it is possible to compute VIX also by integrating suitably modified Black-Scholes implied volatilities.

The SKEW Index is based on the computation of the risk neutral price of a cubic contract on the logreturn of the underlying asset, given by

$$
S=\mathbb{E}_{Q}\left[\left(\frac{\ln \frac{S_{T}}{S_{t}}-\mu}{\sigma}\right)^{3}\right]
$$

Similarly to VIX, it is computed by the quotations of all OTM call and put. It is invariant by translation and scaling and is a pure measure of asymmetry of the risk neutral distribution.

Implicit VaR and Expected Shortfall have been introduced in Barone Adesi (2016). Letting

$$
F_{\mathbb{Q}}(K)=\mathbb{Q}\left(S_{T} \leq K\right) \text { and } f_{\mathbb{Q}}(K)=d F_{\mathbb{Q}} / d K
$$

since it is well known that

$$
\frac{d P(K)}{d K}=e^{-r T} F_{\mathbb{Q}}(K),
$$

the $\tau$-quantile of the risk neutral distribution can be computed by interpolating the function $P(K)$ from the quotations $P\left(K_{i}\right)$, numerically determining $d P(K) / d K$ and finding $\bar{K}$ such that

$$
\left.\frac{d P(K)}{d K}\right|_{K=\bar{K}}=e^{-r T} \tau
$$

The corresponding implicit Expected Shortfall at level $\tau$ is given by

$$
E S_{\tau}=\frac{e^{r T} P(\bar{K})}{\tau}+\bar{K}
$$

Similarly to implicit expectiles and in contrast with VIX and SKEW, the computation of implicit VaR do not require the quotations of all available OTM options. However, it is sensitive to the chosen interpolation method for $P(K)$. 


\section{$3 \quad$ Interexpectile difference}

Expectiles may be interpreted as measures of location, since they satisfy the additivity, homogeneity and monotonicity properties recalled in Proposition 2.1. In order to compute a measure of variability from implicit expectiles, we suggest to consider the interexpectile difference $\Delta_{\tau}$, defined as follows:

$$
\Delta_{\tau}=e_{\tau}(X)-e_{1-\tau}(X)=e_{\tau}(X)+e_{\tau}(-X),
$$

for $X \in L^{1}$ and $\tau \in(1 / 2,1)$. The following properties can be proved straightforwardly from the corresponding properties of expectiles:

Proposition 3.1. Let $X \in L^{1}, \tau \in(1 / 2,1)$ and $\Delta_{\tau}$ as in (7). Then:

a) $\Delta_{\tau}(X) \geq 0$ and $\Delta_{\tau}(X)=0$ if and only if $X=c P$-a.s.

b) $\Delta_{\tau}(X)=\Delta_{\tau}(-X)$

c) $\Delta_{\tau}(X)$ is strictly increasing and continuous in $\tau$

d) $\Delta_{\tau}(X) \rightarrow 0^{+}$for $\tau \rightarrow 1 / 2^{+}$and $\Delta_{\tau}(X) \rightarrow \operatorname{esssup}(X)-\operatorname{essinf}(X)$ for $\tau \rightarrow 1^{-}$

e) $\Delta_{\tau}(X+h)=\Delta_{\tau}(X)$, for $h \in \mathbb{R}$

f) $\Delta_{\tau}(\lambda X)=\lambda \Delta_{\tau}(X)$, for $\lambda \geq 0$

g) $\Delta_{\tau}(X+Y) \leq \Delta_{\tau}(X)+\Delta_{\tau}(Y)$

h) $X \leq_{c x} Y \Rightarrow \Delta_{\tau}(X) \leq \Delta_{\tau}(Y)$,

where $\leq_{c x}$ denotes the usual convex order.

The interexpectile difference is a natural analog to the interquantile difference, that is widely used in exploratory data analysis. It has the advantage of being a true measure of variability, since it is consistent with the convex order: the more disperse a distribution around the mean, the higher are all its interexpectile differences. Moreover, from a), b), e), f) and g) in the previous Proposition it follows that $\Delta_{\tau}$ is a generalized deviation measure in the sense of Rockafellar et al. (2006). We leave further study of its properties to subsequent work; here we illustrate its behaviour on a couple of examples. Let $X$ be distributed as a Bernoulli with parameter $p$. Then by a straightforward computation

$$
\Delta_{\tau}(X)=\frac{\tau p}{\tau p+(1-\tau)(1-p)}-\frac{(1-\tau) p}{(1-\tau) p+\tau(1-p)} .
$$

Let now $X$ be distributed as a standard normal. Here, $\Delta_{\tau}$ does not admit a closed formula and has to be computed by a numerical computation of the expectiles, that can be done e.g. by the $\mathrm{R}$ function enorm. The results are reported in Figure 3. In the Bernoulli case, notice that for each $\tau$ the interexpectile difference is maximal when $p=0.5$, as it happens for the variance. In the normal case, to give a rough rule of the thumb for comparing interexpectile difference and standard deviation, it is possible to compute that $\Delta_{\tau}(X)=\sigma(X)=1$ when $\tau \approx 0.78$. From a financial point of view, interexpectile differences are differences of forward prices of asymmetric forward contracts; in contrast to VIX, they are not prices of portfolios of options.

\section{Empirical results}

In this section we present an empirical analysis of implicit expectiles and interexpectile differences computed on a dataset of closing prices of all quoted FTSE MIB index options from January 2, 2008 to December 30, 2009, kindly provided to us by Borsa Italiana SPA. Data for the underlying FTSE 

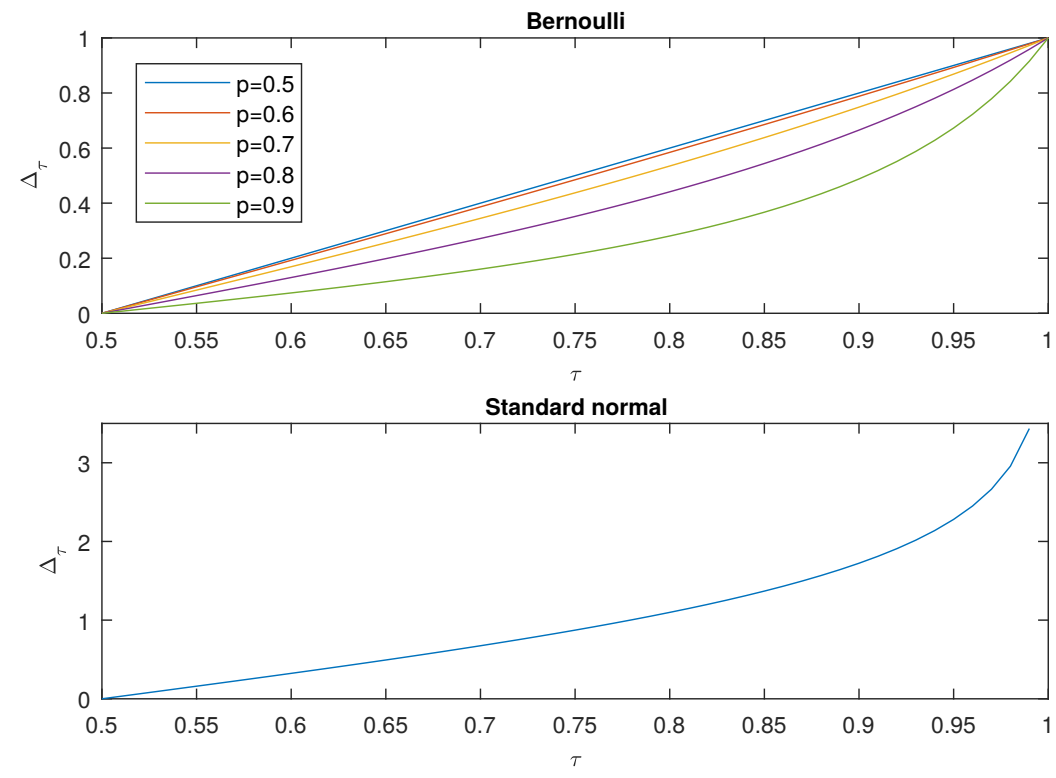

Figure 3: Interexpectile differences as a function of $\tau$ for Bernoulli random variables with $p=$ $0.5,0.6,0.7,0.8,0.9$ (upper panel) and for a standard normal (lower panel).

MIB index, dividend yields and Euribor rates have been downloaded from Bloomberg. It is noted that this period coincides with the U.S. credit and financial crisis, to which European equity markets have typically reacted with significant drops in equity prices and higher volatility. As it is customary in the literature, we adjusted the FTSE MIB index $S_{t}$ for dividends and checked for the no arbitrage conditions given by the Merton bounds

$$
\begin{gathered}
C_{t, T} \geq \max \left(S_{t} e^{-d_{t}(T-t)}-K e^{-r(T-t)}, 0\right) \\
P_{t, T} \geq \max \left(K e^{-r(T-t)}-S_{t} e^{-d_{t}(T-t)}, 0\right),
\end{gathered}
$$

where $d_{t}$ is the yearly continuously compounded dividend yield at time $t$. The number of quoted options on the second available maturity was typically quite low in the considered period, so we decided to limit ourselves only to the first available maturity, with a number of days-to-maturity oscillating between 10 and 36. We report in Figure 4 the strikes for which quotations of call options and put options are available, and in Figure 5 their number, which is on average around 10.

As a first exploratory analysis, we compute inverse expectile curves with the methodology suggested in Section 2, by means of equation (6). In Figure 6 we plot the resulting curves of four typical randomly chosen days. We see that the interexpectile curves are S-shaped and their degree of flatness is related to the number of days-to-maturity and to the volatility level. They are also very close to the theoretical curves of the Black-Scholes model, since the dataset is composed of options with very short maturities. In Figure 7 we show the dynamics of the inverse expectile curves over the whole dataset. From a qualitative point of view, we see that the domain changes according to the movements of the underlying asset, while the inclination is roughly inversely proportional to the volatility level.

The second step of our analysis is the computation of the interexpectile differences $\Delta_{\tau}$. Since the number of quoted options in the dataset is in general quite low, we decided to follow the approach based on the interpolation of implied volatility. For the sake of simplicity we have chosen the quadratic interpolation method of Shimko (1993), that is

$$
\sigma(K)=a_{0}+a_{1} K+a_{2} K^{2} .
$$




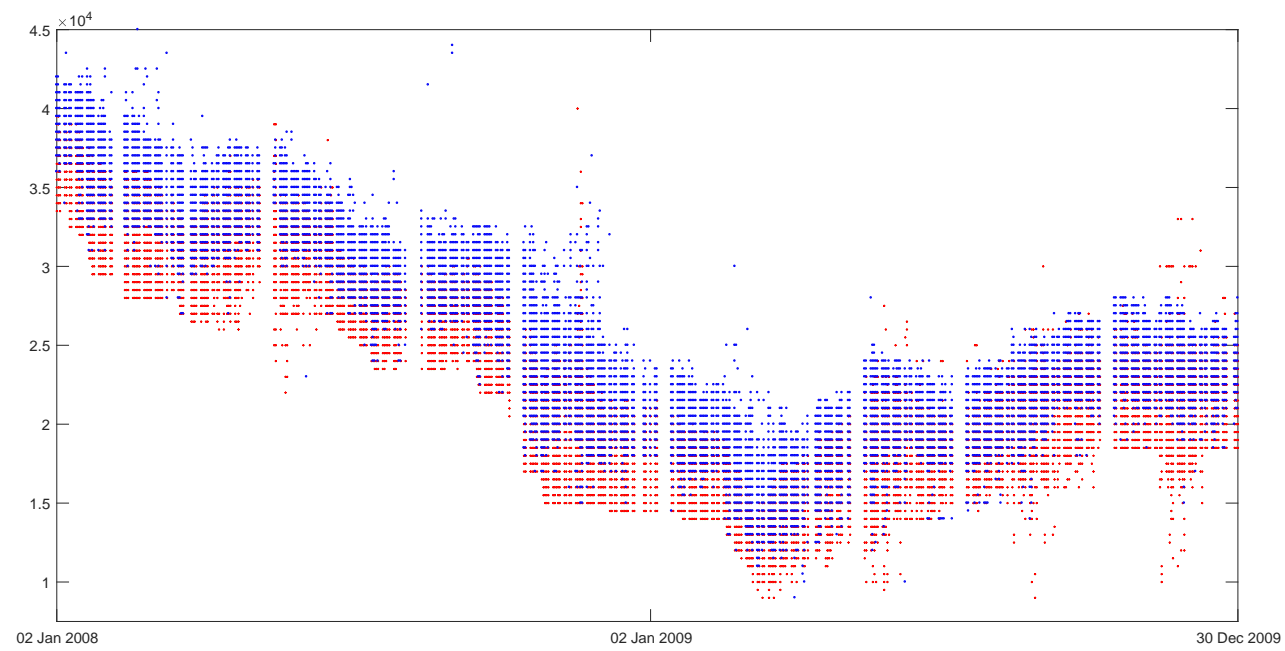

Figure 4: Strike prices for which quotations of call (blue) and put (red) are available as a function of time.

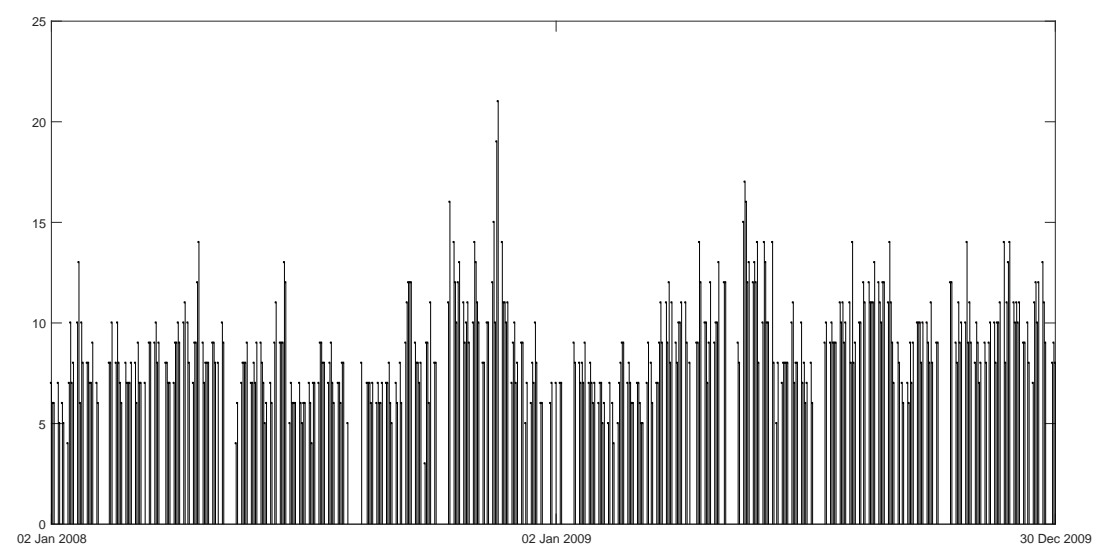

Figure 5: Number of strikes for which quotations of both call and put are available as a function of time. 

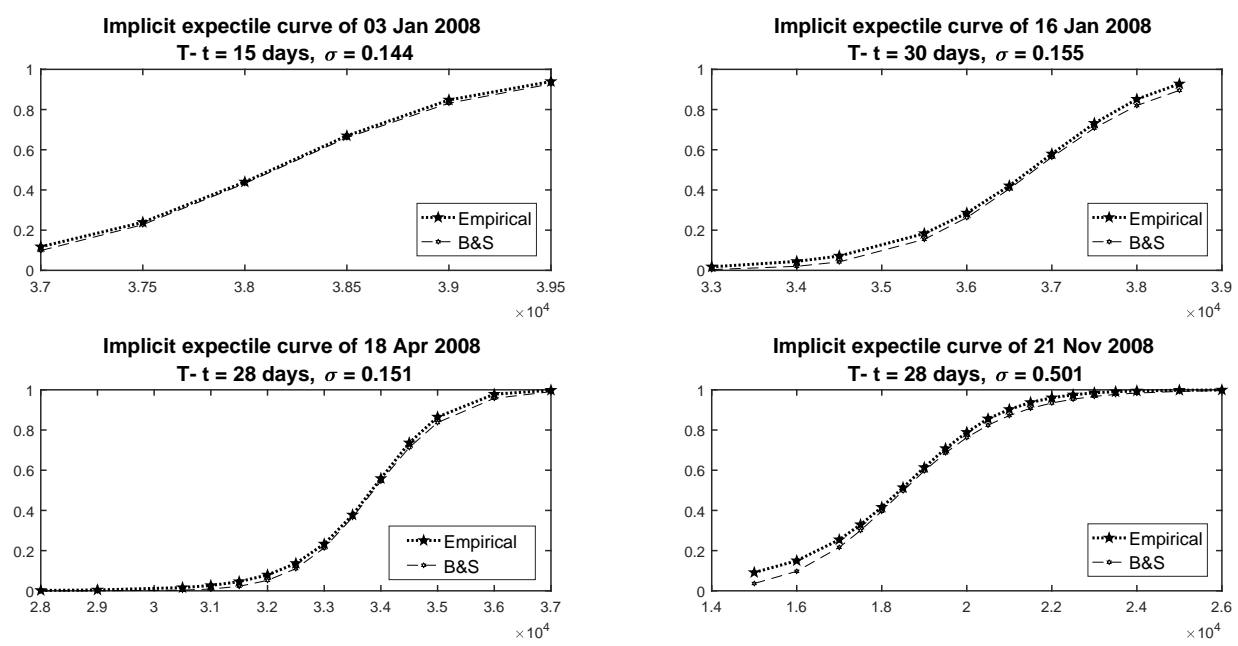

Figure 6: Inverse expectile curves for 03 Jan 2008, 16 Jan 2008, 18 Apr 2008 and 21 Nov 2008, compared with the corresponding curves of the Black-Scholes model.

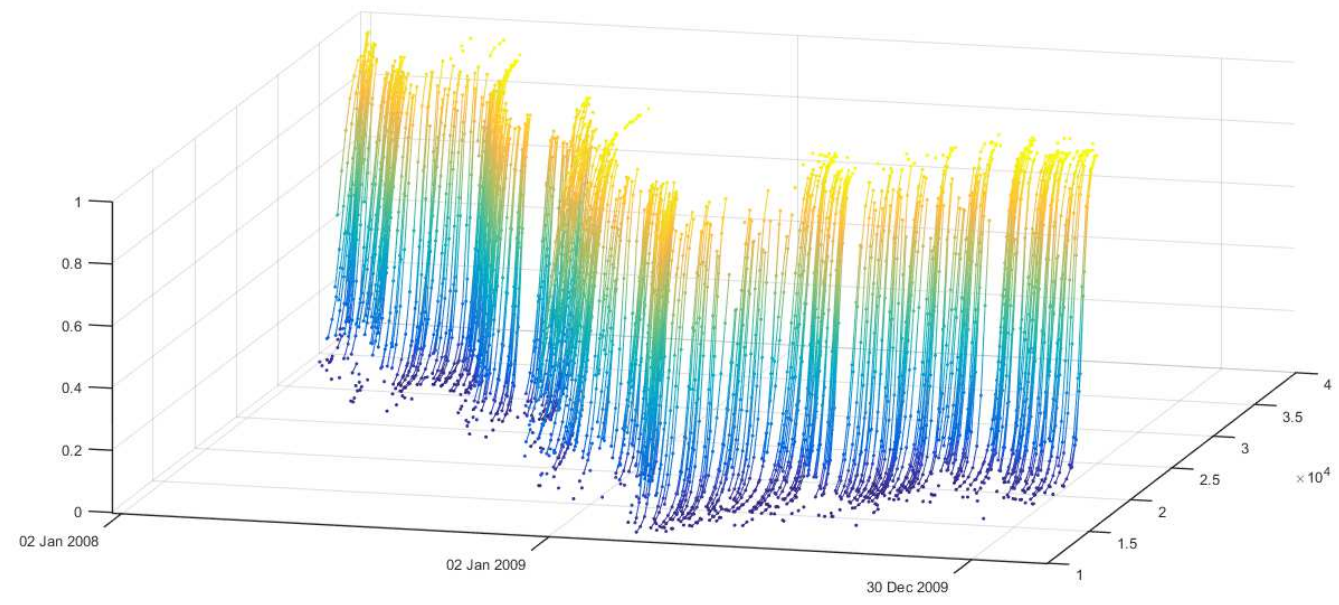

Figure 7: Inverse expectile curves of the whole 2008-2009 dataset. 
The obtained implied volatilities have been used to compute theoretical option prices for all strikes, that enabled us to numerically compute interexpectile differences. The time series of $\Delta_{0.75}$ is displayed in the upper part of Figure 8. The saw-teeth pattern reflects perfectly the number of days-to-maturity, that is varying from 10 to 36, as shown in the lower part of Figure 8. In order to rescale interexpectile
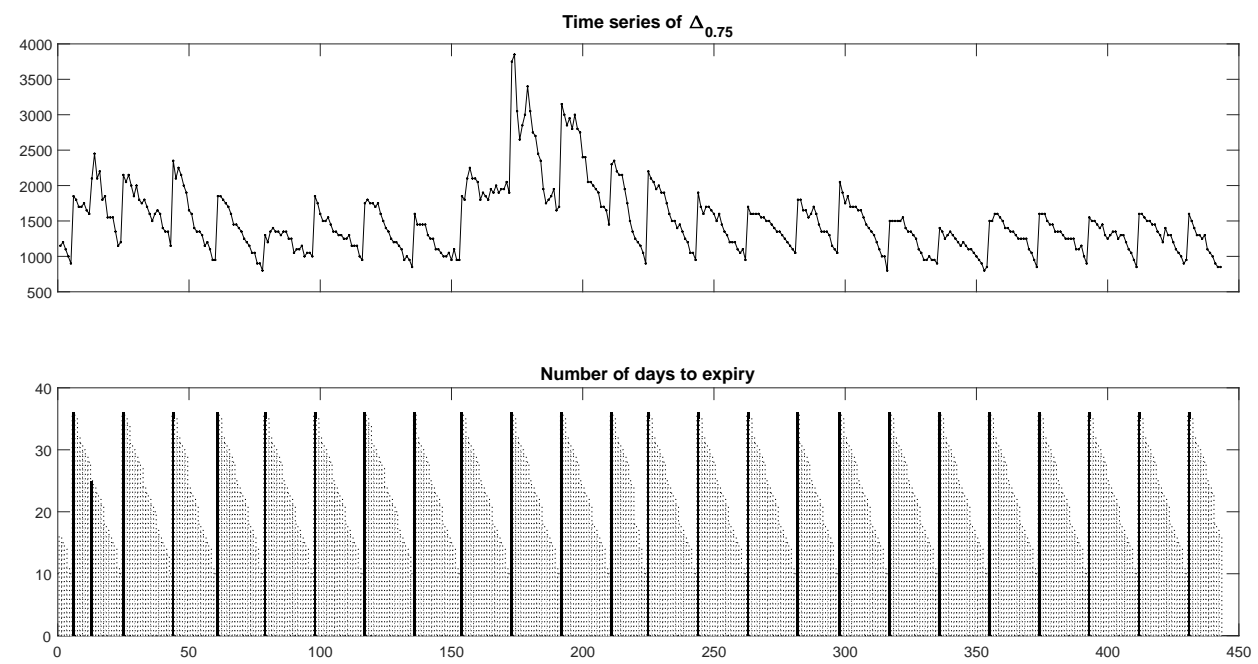

Figure 8: Time series of interexpectile difference $\Delta_{0.75}$ (upper plot) and the corresponding number of days-to-maturity of the options used for its computation (lower plot).

differences to a reference time-to-maturity of 30 days, from now on we set

$$
\Delta_{\tau} \mapsto \Delta_{\tau} \sqrt{\frac{30}{T}}
$$

since $\Delta_{\tau}$ is positively homogeneous and scales like the standard deviation. In order to compare the interexpectile differences with the VIX Index, we compute on our dataset a VIX-like italian volatility index that we call simply IVI Index. In contrast with the standard VIX methodology, since liquidity on the second maturity is very low, we use only quotations for the first maturity and standardize to a 30 days time horizon by the usual square root rule. In Figure 9 we report the empirical interexpectile differences for $\tau=0.60,0.75,0.90$ and the IVI Index; their descriptive statistics are reported in Table 1. Qualitatively, interexpectile differences for different $\tau$ tend to move together and resemble quite well the IVI index, as it is confirmed by the scatterplots in Figure 10 and by the correlation matrix in Table 2. Evidence that interexpectile differences for various $\tau$ tend to move together suggests that the dynamics of the risk neutral distribution are governed by parallel shifts in implicit expectiles. However, further analysis is needed before drawing strong conclusions.

In order to further investigate the dependence structure of interexpectile differences we performed a Principal Component Analysis (PCA) on the time series of $\Delta_{0.60}, \Delta_{0.70}, \Delta_{0.75}, \Delta_{0.80}$ and $\Delta_{0.90}$ (see e.g. Jolliffe (2002)). The time series of the first three principal components are reported in Figure 11, while the mixing matrix and the cumulative fractions of explained variance are reported in Table 3. We see that the first principal component has positive weights and explains more than $98 \%$ of the variability, confirming our findings that interexpectile differences for different $\tau$ tend to move together.

We investigate the stationarity of implicit expectiles and of interexpectile differences by means of the ADF test, implemented in the R package urca (see for more details Dickey and Fuller (1979), 

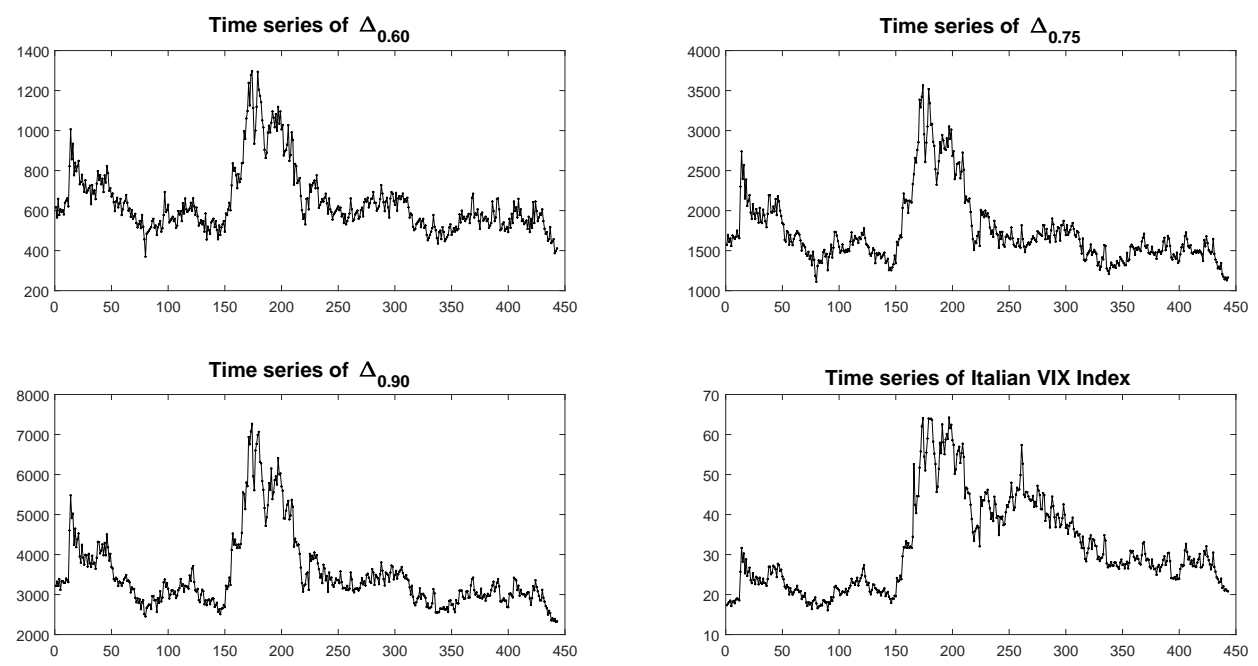

Figure 9: Time series of interexpectile differences $\Delta_{\tau}$ for different $\tau$ and IVI index.

\begin{tabular}{lrrr}
\hline & $\Delta_{0.60}$ & $\Delta_{0.75}$ & $\Delta_{0.90}$ \\
\hline Mean & $6.441 \mathrm{e}+02$ & $1.745 \mathrm{e}+03$ & $3.509 \mathrm{e}+03$ \\
Median & $6.000 \mathrm{e}+02$ & $1.626 \mathrm{e}+03$ & $3.227 \mathrm{e}+03$ \\
Min & $3.700 \mathrm{e}+02$ & $1.111 \mathrm{e}+03$ & $2.325 \mathrm{e}+03$ \\
Max & $1.296 \mathrm{e}+03$ & $3.564 \mathrm{e}+03$ & $7.268 \mathrm{e}+03$ \\
Std. Dev. & $1.623 \mathrm{e}+02$ & $4.412 \mathrm{e}+02$ & $9.245 \mathrm{e}+02$ \\
Skewness & 1.673 & 1.753 & 1.849 \\
Kurtosis & 5.843 & 6.023 & 6.327 \\
\hline
\end{tabular}

Table 1: Descriptive statistics of interexpectile differences $\Delta_{\tau}$ for different $\tau$ and IVI Index.

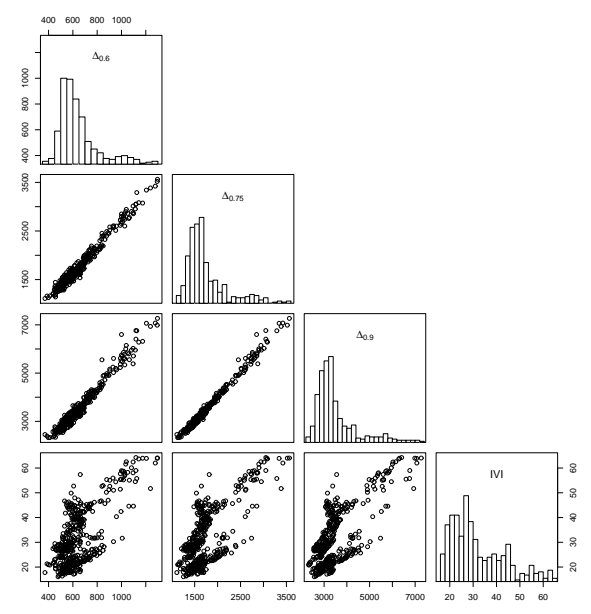

Figure 10: Empirical distribution of interexpectile differences for different $\tau$ and IVI index (main diagonal). Bivariate joint distributions of interexpectile differences for different $\tau$ and IVI index (quadrants off the main diagonal). 


\begin{tabular}{lrrrr}
\hline & $\Delta_{0.60}$ & $\Delta_{0.75}$ & $\Delta_{0.90}$ & IVI \\
\hline$\Delta_{0.60}$ & 1 & & & \\
$\Delta_{0.75}$ & 0.9858 & 1 & & \\
$\Delta_{0.90}$ & 0.9633 & 0.9737 & 1 & \\
IVI & 0.7056 & 0.7208 & 0.7124 & 1 \\
\hline
\end{tabular}

Table 2: Correlation matrix of interexpectile differences for different $\tau$ and IVI index.
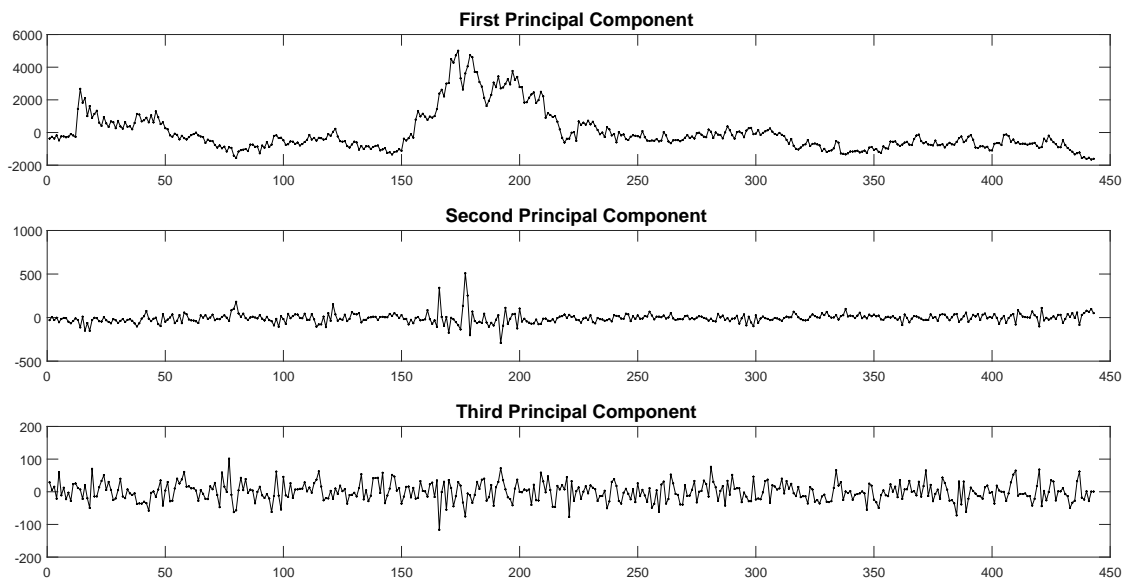

Figure 11: Time series of the first principal components of the interexpectile differences $\Delta_{0.60}, \Delta_{0.70}$, $\Delta_{0.75}, \Delta_{0.80}$ and $\Delta_{0.90}$.

\begin{tabular}{ccccc}
\hline \multicolumn{5}{c}{ Mixing matrix } \\
\hline 0.1294 & -0.1521 & 0.4109 & -0.4002 & 0.7944 \\
0.2738 & -0.3272 & 0.6656 & -0.2300 & -0.5674 \\
0.3555 & -0.4181 & 0.1055 & 0.8019 & 0.2114 \\
0.4521 & -0.5220 & -0.6139 & -0.3794 & -0.0472 \\
0.7599 & 0.6500 & 0.0600 & 0.0016 & -0.0017 \\
\hline \hline \multicolumn{5}{c}{ Cumulative explained variance } \\
\hline 0.9865 & 0.9986 & 0.9992 & 0.9996 & 1.0000 \\
\hline
\end{tabular}

Table 3: Weights of the principal components of the interexpectile differences $\Delta_{0.60}, \Delta_{0.70}, \Delta_{0.75}$, $\Delta_{0.80}$ and $\Delta_{0.90}$ (upper panel) and cumulative fraction of explained variance (lower panel). 
Pfaff (2008)). The ADF test assumes that the time series $y_{t}$ under scrutiny has the dynamics

$$
\Delta y_{t}=\alpha_{0}+\alpha_{1} t+\gamma y_{t-1}+\sum_{i=1}^{p} \delta_{i} \Delta y_{t-i}+u_{t},
$$

where $p$ is the order of the autoregressive process and $u_{t}$ is a residual term, and tests the null hypothesis $\left\{H_{0}: \gamma=0\right\}$ versus the alternative $\left\{H_{1}: \gamma<0\right\}$. The lag order $p$ is chosen in order to guarantee that the residuals $u_{t}$ are uncorrelated, by means of the procedure suggested in $\mathrm{Ng}$ and Perron (1995), that is based on the optimal Akaike Information Criterion. In Table 4 we report the estimated values of $\gamma$ and the corresponding ADF test statistic for levels and time differences of $e_{\tau}$ and $\Delta_{\tau}$. The results show that in all cases nonstationarity of the considered series of $e_{\tau}$ and $\Delta_{\tau}$ cannot be rejected at the $10 \%$ level, while nonstationarity of time differences of $e_{\tau}$ and $\Delta_{\tau}$ is always rejected at the $1 \%$ level. We conclude that in our dataset implicit expectiles and interexpectiles are not stationary, while their time differences are stationary.

\begin{tabular}{|c|c|c|c|c|c|c|}
\hline \multicolumn{7}{|c|}{ Results of the ADF test } \\
\hline & \multicolumn{2}{|c|}{$e_{0.10}$} & \multicolumn{2}{|c|}{$e_{0.25}$} & \multicolumn{2}{|c|}{$e_{0.40}$} \\
\hline & levels & time diff. & levels & time diff. & levels & time diff. \\
\hline$\hat{\gamma}$ & -0.0059 & -1.2095 & -0.0056 & -1.0530 & -0.0049 & -1.0700 \\
\hline t-test & -0.8290 & -4.9570 & -0.9009 & -14.859 & -0.8270 & -15.035 \\
\hline \multicolumn{3}{|c|}{$e_{0.60}$} & \multicolumn{2}{|c|}{$e_{0.75}$} & \multicolumn{2}{|c|}{$e_{0.90}$} \\
\hline & levels & time diff. & levels & time diff. & levels & time diff. \\
\hline$\hat{\gamma}$ & -0.0045 & -1.1046 & -0.0044 & -1.1328 & -0.0048 & -1.1495 \\
\hline t-test & -0.7840 & -15.427 & -0.7732 & -15.644 & -0.8170 & -10.395 \\
\hline \multicolumn{3}{|c|}{$\Delta_{0.60}$} & \multicolumn{2}{|c|}{$\Delta_{0.75}$} & \multicolumn{2}{|c|}{$\Delta_{0.90}$} \\
\hline & levels & time diff. & levels & time diff. & levels & time diff. \\
\hline$\hat{\gamma}$ & -0.0388 & -1.5317 & -0.0369 & -1.4098 & -0.0325 & -1.2462 \\
\hline t-test & -2.2970 & -15.087 & -2.6590 & -12.664 & -2.4400 & -16.917 \\
\hline
\end{tabular}

Table 4: Estimated parameters $\hat{\gamma}$ and corresponding ADF test t-statistic for levels and time differences of implicit expectiles $e_{\tau}$ and changes in interexpectile differences $\Delta_{\tau}$. The critical values are $c_{1 \%}=$ $-3.98, c_{5} \%=-3.42$ and $c_{10 \%}=-3.13$.

\section{Modelling interexpectile differences with continuous autore- gressive models}

Continuous-time models allow to manage irregularly spaced data, for example due to weekends or holidays, without considering any missing data imputation technique. Figure 4 suggests some irregularity in the time distribution of observations in our dataset.

In this section, we investigate the autoregressive structure of the variability measure $\Delta_{\tau}$. In particular, we model the logarithm of the interexpectile difference index as a Lévy Continuous Autoregressive model of order $1, \operatorname{CAR}(1)$ hereafter, with location parameter $\mu$ whose dynamics is described by the two following equations:

$$
\begin{aligned}
Y_{t} & =\mu+b_{0} X_{t} \\
\mathrm{~d} X_{t} & =-a_{1} X_{t} \mathrm{~d} t+\mathrm{d} L_{t} .
\end{aligned}
$$

Notice that if we fix $\mu=0$ and $b_{0}=1$, we have as a special case the Lévy Ornstein-Uhlenbeck that has been extensively used for volatility modelling (see Barndorff-Nielsen and Shephard, 2001, for example). For the Lévy term $L_{t}$ two infinitely divisible distributions are considered: Variance 
Gamma (VG) and Normal Inverse Gaussian (NIG) both with the parametrization as in Iacus and Mercuri (2015). Estimation is performed using the R package YUIMA (see Brouste et al., 2014) that, as described in Iacus and Mercuri (2015), requires three steps. First we estimate the CAR(1) parameters $\left(\mu, a_{1}, b_{0}\right)$, then we extract the Lévy increments and finally we estimate the Lévy measure parameters. Fitted CAR(1) parameters for the $\log$ of interexpectile differences for $\tau=0.50,0.75,0.90$ are reported in Table 5. Parameters of a VG fitted to the noise term are reported in Table 6 while fitted NIG parameters in Table 7. Based on the log-likelihood, we can affirm that the CAR(1) with NIG increments displays a better fit than the model with VG distributed increments. It is interesting to notice the differences in Figure 12 in terms of asymmetry for the distribution of increments as $\tau$ varies.

\begin{tabular}{lcccccc}
\hline \multicolumn{6}{c}{ CAR(1) estimated parameters } \\
\hline \multicolumn{4}{c}{$\Delta_{0.60}$} & \multicolumn{2}{c}{$\Delta_{0.75}$} & \multicolumn{2}{c}{$\Delta_{0.90}$} \\
Parameter & Estimate & s.d. & Estimate & s.d. & Estimate & s.d. \\
\hline$b_{0}$ & 1.355 & $(0.047)$ & 2.672 & $(0.097)$ & -1.007 & $(0.033)$ \\
$a_{1}$ & 18.210 & $(4.719)$ & 43.535 & $(7.719)$ & 0.036 & $(\mathrm{NaN})$ \\
$\mu$ & 6.428 & $(0.055)$ & 7.264 & $(0.045)$ & 2.330 & $(\mathrm{NaN})$ \\
\hline$-2 \operatorname{LogL}$ & -952.701 & -394.412 & -1166.671 \\
\hline
\end{tabular}

Table 5: Fitted parameters with corresponding standard error (s.d.) for a CAR(1) model used for modelling $\log \Delta_{\tau}$.

\begin{tabular}{|c|c|c|c|c|c|c|}
\hline \multicolumn{7}{|c|}{ VG estimated parameters } \\
\hline \multirow[b]{2}{*}{ Parameter } & \multicolumn{2}{|c|}{$\Delta_{0.60}$} & \multicolumn{2}{|c|}{$\Delta_{0.75}$} & \multicolumn{2}{|c|}{$\Delta_{0.90}$} \\
\hline & Estimate & s.d. & Estimate & s.d. & Estimate & s.d. \\
\hline$\lambda$ & 11.9982 & $(194.123)$ & 0.526 & $(\mathrm{NaN})$ & 2.088 & $(9.640)$ \\
\hline$\alpha$ & 4.3430 & $(2.354)$ & 0.471 & $(0.024)$ & 31.981 & $(5.432)$ \\
\hline$\beta$ & 0.6711 & $(0.797)$ & 0.082 & $(0.023)$ & -3.047 & $(2.398)$ \\
\hline$\mu_{0}$ & -0.8740 & $(0.973)$ & -0.385 & $(0.007)$ & 0.012 & $(0.143)$ \\
\hline$-2 \log \mathrm{L}$ & \multicolumn{2}{|c|}{1389.589} & \multicolumn{2}{|c|}{1780.248} & \multicolumn{2}{|c|}{-1184.214} \\
\hline
\end{tabular}

Table 6: Estimated VG parameters with corresponding standard error (s.d.) for the noise in a CAR(1) model used for modelling $\log \Delta_{\tau}$.

\begin{tabular}{lcccccc}
\hline \multicolumn{7}{c}{ NIG estimated parameters } \\
\hline \multicolumn{2}{c}{$\Delta_{0.6}$} & \multicolumn{2}{c}{$\Delta_{0.75}$} & \multicolumn{2}{c}{$\Delta_{0.90}$} \\
Parameter & Estimate & s.d. & Estimate & s.d. & Estimate & s.d. \\
\hline$\alpha$ & 3.031 & $(1.939)$ & 0.299 & $(0.065)$ & 19.299 & $(4.257)$ \\
$\beta$ & 0.647 & $(0.828)$ & 0.184 & $(0.050)$ & -3.462 & $(2.227)$ \\
$\delta_{1}$ & 3.866 & $(35.160)$ & 0.998 & $(1.356)$ & 0.078 & $(0.208)$ \\
$\mu_{0}$ & -0.844 & $(16.174)$ & -0.765 & $(1.517)$ & 0.014 & $(0.128)$ \\
\hline-2 LogL & \multicolumn{2}{c}{1389.623} & 1728.580 & -1187.290 \\
\hline
\end{tabular}

Table 7: Estimated VG parameters with corresponding standard error (s.d.) for the noise in a CAR(1) model used for modelling $\log \Delta_{\tau}$. 


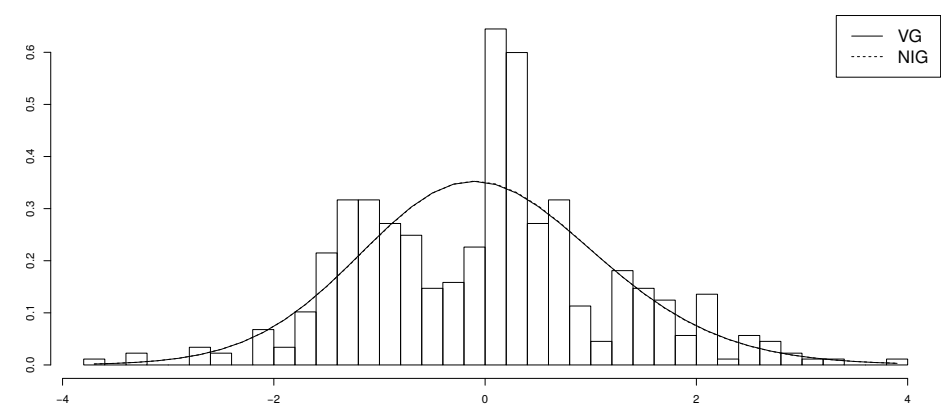

(a) $\Delta_{0.60}$

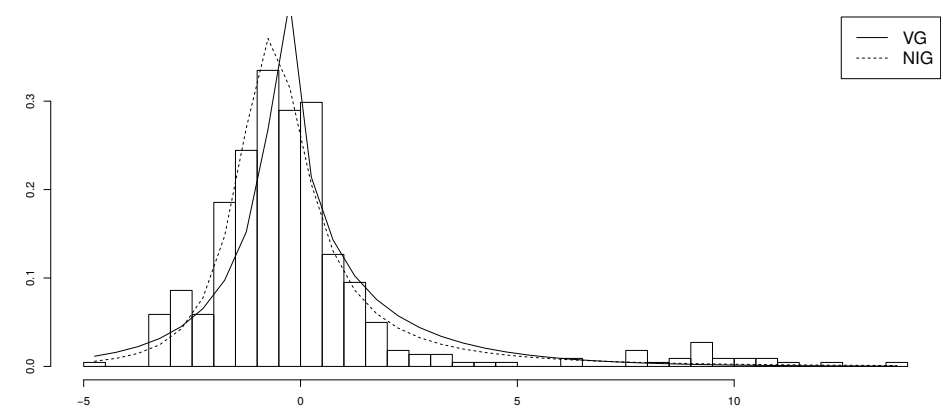

(b) $\Delta_{0.75}$

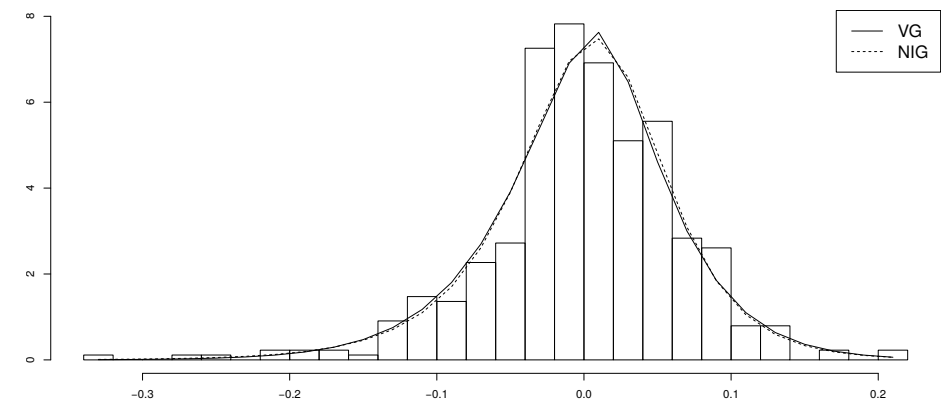

(c) $\Delta_{0.90}$

Figure 12: Fitted densities of increments using a CAR(1) model with VG and NIG innovations. 


\section{$6 \quad$ Forecasting power of implicit expectiles and interexpectile differences}

There is a general agreement in the literature about a negative correlation between contemporaneous variations of the VIX index and returns of the S\&P500 Index, a stylized fact known as leverage effect. In our dataset, interexpectile differences display a similar pattern, as reported in Figure 13. Several studies (see Giot (2005), Banerjee et al. (2007), Rubbaniy et al. (2014), Elyasiani et al. (2016)) considered the forecasting power of volatility indexes (either their levels, or their time differences) on future returns. Following the methodology of Banerjee et al. (2007), Rubbaniy et al. (2014), Elyasiani
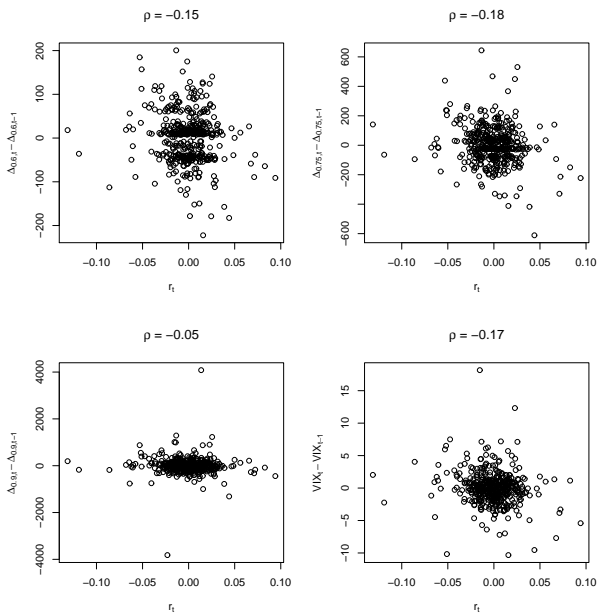

Figure 13: Joint distributions of changes in interexpectile differences and time differences of the IVI index (vertical axis) versus contemporaneous FTSE MIB returns (horizontal axis).

et al. (2016), we consider the regression

$$
R_{t, t+\Delta t}=\alpha+\beta I_{t}+\hat{\epsilon}_{t}
$$

where $R_{t, t+\Delta t}$ is the FTSE MIB index log return on the time interval $(t, t+\Delta t)$ and $I_{t}$ is the time series of the variable whose forecasting power is under examination. In the literature, both the levels of VIX and the time differences of VIX have been used in place of $I_{t}$ in the forecasting regression (12). Giot (2005) studied the relationship between the levels of (old) VIX and VXN and the future returns on respectively the S\&P100 and the NASDAQ100, finding a moderate evidence that high levels of VIX and VXN predict positive future returns. Banerjee et al. (2007) considered the forecasting power on future returns of both levels and differences of VIX, finding that the VIX level has a significative forecasting power at the $5 \%$ level, while VIX differences have no correlations with future returns. Rubbaniy et al. (2014) considered VIX, VXN and VDAX and found some evidence that the levels of the volatility indexes have some forecasting power to 20-day and 60-day returns.

In Table 8 we report the results of the estimation of (12) when the forecasting variable $I_{t}$ are the levels of $\Delta_{0.75}$, IVI Index, $e_{0.25}$ and $e_{0.75}$. In Table 9 we report the results of the estimation of (12) for the series of time differences of the previous quantities. As it is customary in the literature, we used NeweyWest estimators to overcome the problems of autocorrelation of residuals and heteroscedasticity. In the first column of Table 8 , the variable $\Delta_{0.75}$ has some predictive power for future returns up to 60 days; the predictive power seems slightly superior than the predictive power of the IVI Index reported in the second column. The levels of implicit expectiles do not seem to have predictive power at any return horizon. In Table 9 the situation is less clear; the series of the time differences of all the considered quantities seem to have some predictive power for future returns up to 60 days. These 
findings are consistent with Banerjee et al. (2007) and provide, in our opinion, further motivation for the study of implicit expectiles and alternative measures of implied volatility.

\begin{tabular}{c|lcccc}
\hline & & $\Delta_{0.75}$ & IVI & $e_{0.25}$ & $e_{0.75}$ \\
\hline$R_{t, t+1}$ & $\hat{\beta}$ & $-7.713 \mathrm{e}-06^{*}(-2.068)$ & $-1.727 \mathrm{e}-04(-1.360)$ & $2.147 \mathrm{e}-09(0.012)$ & $-3.824 \mathrm{e}-08(-0.219)$ \\
& $R^{2}$ & $2.18 \%$ & $0.75 \%$ & $3.32 \mathrm{e}-05 \%$ & $0.01 \%$ \\
\hline$R_{t, t+7}$ & $\hat{\beta}$ & $-3.683 \mathrm{e}-05^{* *}(-3.220)$ & $-9.704 \mathrm{e}-04(-1.540)$ & $4.128 \mathrm{e}-08(0.038)$ & $-2.601 \mathrm{e}-07(-0.247)$ \\
& $R^{2}$ & $12.85 \%$ & $5.12 \%$ & $2.45 \mathrm{e}-03 \%$ & $0.09 \%$ \\
\hline$R_{t, t+30}$ & $\hat{\beta}$ & $-1.401 \mathrm{e}-04^{* * *}(-9.154)$ & $-3.711 \mathrm{e}-03^{* * *}(-4.936)$ & $1.204 \mathrm{e}-06(0.468)$ & $3.205 \mathrm{e}-07(0.135)$ \\
& $R^{2}$ & $26.50 \%$ & $11.40 \%$ & $0.32 \%$ & $0.02 \%$ \\
\hline$R_{t, t+60}$ & $\hat{\beta}$ & $-2.307 \mathrm{e}-04^{* * *}(-10.885)$ & $-9.253 \mathrm{e}-03^{* * *}(-5.422)$ & $7.595 \mathrm{e}-06(1.331)$ & $4.651 \mathrm{e}-06(0.844)$ \\
& $R^{2}$ & $38.62 \%$ & $32.26 \%$ & $3.99 \%$ & $1.45 \%$ \\
\hline$R_{t, t+90}$ & $\hat{\beta}$ & $-4.034 \mathrm{e}-04(-0.308)$ & $-9.919 \mathrm{e}-03(-1.525)$ & $3.005 \mathrm{e}-06(0.053)$ & $1.314 \mathrm{e}-06(0.020)$ \\
& $R^{2}$ & $35.25 \%$ & $24.94 \%$ & $0.68 \%$ & $0.12 \%$ \\
\hline
\end{tabular}

Table 8: Estimated parameters for the regression (12), with corresponding $t$ statistics in brackets, using respectively as forecasting variables $I_{t}$ the levels of $\Delta_{0.75}$, IVI Index, $e_{0.25}$ and $e_{0.75}$.

\begin{tabular}{|c|c|c|c|c|c|}
\hline & & time diff. $\Delta_{0.75}$ & time diff. IVI & time diff. $e_{0.25}$ & time diff. $e_{0.75}$ \\
\hline \multirow[t]{2}{*}{$R_{t, t+1}$} & $\hat{\beta}$ & $-3.233 \mathrm{e}-05^{* * *}(-3.561)$ & $-1.465 \mathrm{e}-03^{*}(-2.126)$ & $-6.947 \mathrm{e}-07(-0.495)$ & $-3.791 \mathrm{e}-06(-1.845)$ \\
\hline & $R^{2}$ & $3.23 \%$ & $2.64 \%$ & $0.28 \%$ & $0.68 \%$ \\
\hline \multirow[t]{2}{*}{$R_{t, t+7}$} & $\hat{\beta}$ & $-1.030 \mathrm{e}-04^{* * *}(-5.650)$ & $-0.0059271 * * *(-5.396)$ & $3.645 \mathrm{e}-05^{* * *}(9.895)$ & $3.679 \mathrm{e}-05^{* * *}(8.004)$ \\
\hline & $R^{2}$ & $8.85 \%$ & $11.67 \%$ & $21.14 \%$ & $17.39 \%$ \\
\hline \multirow[t]{2}{*}{$R_{t, t+30}$} & $\hat{\beta}$ & $7.616 \mathrm{e}-05^{* *}(2.682)$ & $4.202 \mathrm{e}-03^{* * *} \quad(4.097)$ & $-1.964 \mathrm{e}-05^{* *} \quad(-3.008)$ & $-1.813 \mathrm{e}-05^{*}(-2.264)$ \\
\hline & $R^{2}$ & $1.59 \%$ & $2.03 \%$ & $1.93 \%$ & $1.34 \%$ \\
\hline \multirow[t]{2}{*}{$R_{t, t+60}$} & $\hat{\beta}$ & $1.322 \mathrm{e}-04^{* *}(2.625)$ & $5.678 \mathrm{e}-03^{*}(2.278)$ & $-2.521 \mathrm{e}-05^{*}(-2.553)$ & $-2.377 \mathrm{e}-05^{*}(-2.025)$ \\
\hline & $R^{2}$ & $1.82 \%$ & $1.49 \%$ & $1.60 \%$ & $1.10 \%$ \\
\hline \multirow[t]{2}{*}{$R_{t, t+90}$} & $\hat{\beta}$ & $1.102 \mathrm{e}-04(1.767)$ & $5.256 \mathrm{e}-03(1.893)$ & $-2.373 \mathrm{e}-05^{*}(-2.302)$ & $-2.156 \mathrm{e}-05(-1.948)$ \\
\hline & $R^{2}$ & $0.97 \%$ & $0.93 \%$ & $0.90 \%$ & $0.57 \%$ \\
\hline
\end{tabular}

Table 9: Estimated parameters for the regression (12), with corresponding $t$ statistics in brackets, using respectively as forecasting variables $I_{t}$ the time differences of $\Delta_{0.75}$, IVI Index, $e_{0.25}$ and $e_{0.75}$. 


\section{Conclusions and directions for further research}

We showed that it is straightforward to compute the expectiles of the risk neutral distribution from quoted European option prices. The empirical analysis of implicit expectile curves is quite preliminary but shows the feasibility and the theoretical soundness of the approach. Interexpectile differences are novel measures of variability that arise naturally in this context and have many desirable properties. Their study in the broader context of the theory of variability measures is the subject of undergoing parallel research. From an empirical point of view, although our dataset is quite limited, the results seem to indicate that implicit expectiles and interexpectile differences may complement the wellestablished VIX methodology, and are surely worth of further empirical and theoretical analysis.

\section{References}

Bakshi, G., Kapadia, N. and Madan, D., Stock Return Characteristics, Skew Laws, and the Differential Pricing of Individual Equity. The Review of Financial Studies, 2003, 16, 101-143.

Banerjee, P.S., Doran, J. and Peterson, D., Implied volatility and future portfolio returns. Journal of Banking and Finance, 2007, 31, 3183-3199.

Barndorff-Nielsen, O.E. and Shephard, N., Non-Gaussian Ornstein Uhlenbeck-based models and some of their uses in financial economics. Journal of the Royal Statistical Society: Series B (Statistical Methodology), 2001, 63, 167-241.

Barone Adesi, G., VaR and CVaR implied in option prices. Journal of Risk and Financial Management, 2016, 9.

Barone Adesi, G., Legnazzi, C. and Sala, C., SP500 Index, an Option-Implied Risk Analysis. Working paper SSRN, 2016a.

Barone Adesi, G., Legnazzi, C. and Sala, C., WTI Crude Oil Option-Implied VaR and CVaR: An Empirical Application. Working paper SSRN, 2016b.

Bellini, F. and Bignozzi, V., On elicitable risk measures. Quantitative Finance, 2015, 15, 725-733.

Bellini, F. and Di Bernardino, E., Risk management with expectiles. The European Journal of Finance, 2017, 23, 487-506.

Bellini, F., Klar, B., Müller, A. and Rosazza Gianin, E., Generalized quantiles as risk measures. Insurance: Mathematics and Economics, 2014, 54, 41-48.

Bellini, F., Klar, B. and Müller, A., Expectiles, Omega Ratios and Stochastic Ordering. Methodology and Computing in Applied Probability, 2016 https://doi.org/10.1007/s11009-016-9527-2.

Breeden, D. and Litzenberger, R., Prices of State-Contingent Claims Implicit in Option Prices. Journal of Business, 1978, 55, 839-866.

Britten-Jones, M. and Neuberger, A., Option Prices, Implied Price Processes, and Stochastic Volatility. Journal of Finance, 2000, 51, 621-651.

Brouste, A., Fukasawa, M., Hino, H., Iacus, S.M., K., K., Koike, Y., Masuda, H., Nomura, R., Ogihara, T., Shimuzu, Y., Uchida, M. and Yoshida, N., The YUIMA Project: A Computational Framework for Simulation and Inference of Stochastic Differential Equations. Journal of Statistical Software, $2014, \mathbf{5 7}, 1-51$.

CBOE, The CBOE Volatility Index: VIX. CBOE White Paper, 2009. 
CBOE, The CBOE Skewness Index. CBOE White Paper, 2010.

Delbaen, F., Bellini, F., Bignozzi, V. and Ziegel, J., Risk measures with the CxLS property. Finance and Stochastics, 2016, 20, 433-453.

Demeterfi, K., Derman, E., Kamal, K. and Zou, J., More Than You Ever Wanted To Know About Volatility Swaps. Quantitative Strategies Research Notes Goldman Sachs \&3 Co, 1999.

Dickey, D.A. and Fuller, W.A., Distribution of the estimators for autoregressive time series with a unit root. Journal of the American Statistical Association, 1979, 74, 427-431.

Elyasiani, E., Gambarelli, L. and Muzzioli, S., The Risk-Asymmetry Index. Working Paper SSRN, 2016

Fukasawa, M., Ishida, I., Maghrebi, N., Oya, K., Ubukata, M. and Yamazaki, K., Model-free implied volatility: from surface to index. International Journal of Theoretical and Applied Finance, 2011, 14, 433-463.

Giot, P., Relationships between implied volatility indexes and stock index returns. Journal of Portfolio Management, 2005, 26, 12-17.

Iacus, S.M. and Mercuri, L., Implementation of Lévy CARMA model in Yuima package. Computational Statistics, 2015, 30, 1111-1141.

Jiang, G. and Tian, Y., Extracting model-free volatility from option prices: an estimation of the VIX index. Journal of Derivatives, 2007, 14, 1-26.

Jolliffe, I.T., Principal Component Analysis, 2002 (Springer: New York), ISBN 978-0-387-22440-4.

Newey, W. and Powell, J., Asymmetric least squares estimation and testing. Econometrica, 1987, 55, 819-847.

Ng, S. and Perron, P., Unit root tests in ARMA models with data-dependent methods for the selection of the truncation lag. Journal of the American Statistical Association, 1995, 90, 268-281.

Pfaff, B., Analysis of Integrated and Cointegrated Time Series with R, Second Edition, 2008 (Springer: New York), ISBN 0-387-27960-1.

Rockafellar, R.T., Uryasev, S. and Zabarankin, M., Generalized deviations in risk analysis. Finance and Stochastics, 2006, 10, 51-74.

Rubbaniy, G., Asmerom, R., Rizvi, S.K.A. and Naqvi, B., Do fear indices help predict stock returns?. Quantitative Finance, 2014, 14, 831-847.

Shimko, D., Bounds of probability. Risk, 1993, 6, 33-37. 